\title{
Is there more global solar activity on the Sun?
}

\author{
J. X. Wang ${ }^{1}$, Y. Z. Zhang ${ }^{1}$, G. P. Zhou ${ }^{1}$, Y. Y. Wen ${ }^{1}$ and J. Jiang ${ }^{2}$ \\ ${ }^{1}$ Key Laboratory of Solar Activity, National Astronomical Observatories of China, CAS, \\ A20 Datun Road, Chaoyang District, Beijing, 100012 \\ email: wangjx@ourstar.bao.ac.cn \\ ${ }^{2}$ Max Planck Institute for Solar System Research \\ email: jiangjie@ourstar.bao.ac.cn
}

\begin{abstract}
There appear indications of more global activity on the Sun which is larger, much beyond the scale of solar active regions (ARs). These indications include formation, flaring and eruption of the trans-equatorial loops seen in EUV and X-rays, formation and eruption of transequatorial filaments, global magnetic connectivity in EUV dimming associated with halo-coronal mass ejections, wide spread of radio burst sources in meter wavelength in the solar corona, and quasi-simultaneous magnetic flux emergence in both hemispheres seen during some major solar events. With examples of a few major events in the last solar cycle we discuss the possibility that there is large or global-scale activity on the Sun. Its spatial scale is many times larger than that of AR and temporal scale is over 10 hours. The exemplified trans-equatorial loops are anchored in ARs and their activity is temporally associated with flares in ARs too. In some sense the flares in ARs appear either as a part of or a precursor of the more global activity. It is likely that the combination of the flares in ARs and the associated global activity is responsible to the major solar-terrestrial events. More efforts in understanding the global activity are undertaken.
\end{abstract}

Keywords. Sun: activity, Sun: filaments, Sun: flares, Sun: magnetic fields.

\section{Introduction}

In the discussions of a few Sun-Earth connection events, there appear two extreme viewpoints to be discerned (see Culhane \& Siscoe 2007). On one hand the flare/CME events may be related to quasi-simultaneous magnetic interactions that occur on a global scale given favorable magnetic topology (Wang et al., 2006, 2007; Zhang et al. 2007; Zhou et al., 2007). Alternatively, the eruptions may begin on the scale of an individual AR and then become global in nature owing to mechanisms of the kind described by Attrill et al. (2006), Harra et al. (2007), and Mandrini et al. (2007).

Although some large-scale CMEs are showing global signature on the solar surface, the question raised by van Driel-Gesztelyi et al. (2008) is penetrating: are these events having intrinsically large or global scale in nature or developing from small or AR scale by nurture. As summarized by these authors, some recent studies suggest that those CMEs are inherently in large-scale, so that their energy supply and final angular width originate from a large-scale region (see Zhang et al., 2007; Wen et al. 2007; Wang et al., 2007; Zhou et al. 2007; Zhukov and Veselovsky, 2007); while, other approaches consider that CMEs start from AR scale, and then evolve to become large-scale even in the low corona due to interaction between the expanding magnetic structure of the CMEs and other lowcoronal magnetic structures (see Maia et al., 1999; Pohjolainen et al., 2001; Bemporad et al., 2005; Attrill et al., 2007a; Mandrini et al., 2007; Moore and Sterling, 2007).

Put aside how small the initiation site of a CME is, our questions are: (1) whether or not there is a large- or global-scale magnetic structure which is involved in the CME process? 
(2) Is there more global activity which goes together with flares/filament eruption in AR scale in the CME process?

To be accurate and clear, whenever we say large-scale of the CME source region, we simply mean that the material and magnetic flux of a CME come from global scale coronal structure. A CME may be triggered in an AR or in a filament channel, and in such a "small-scale" complex magnetic structure, it may manifest first as a flare. Moreover, multi-magnetic flux system and complex topology connections may be included in a CME source region, and magnetic interaction among them would, of course, be unavoidable.

In this paper, we exemplify a few major events in the last solar cycle, and try to answer if there are a large-scale magnetic structures involved in flare/CMEs, and if there is more global activity on the Sun which comprises flare and CME as its inseparable manifestation. In section 2 a few flare/CME events are exemplified, and then discussions are given on what we can learn from these observations.

\section{Indications To Global Scale Solar Activity}

\subsection{On the Halloween events in 2003}

Ceaseless magnetic activity happens on the Sun everywhere. However, is there a hierarchy of the Sun's magnetic activity (Zirin, 1985)? Are the different rank magnetic structures in the hierarchy similar? Is the small-scale magnetic activity the indispensable parts of the AR-scale activity? Similarly, is AR-scale activity a part of the larger scale magnetic activity? Do different levels of magnetic activity simultaneously happen or be intercoupled or interacted each other? Whether we pay too more attentions to AR phenomena, or we simply isolate an AR from their large-scale field background (see Liu et al. 2007)? Here, a few major events are used to examine the possibility of large- or global-scale magnetic structures involved in a CME in the Sun's low corona.

The extraordinary episodes in October - November 2003 marked some of the most intense solar activity in the 23rd solar circle, manifesting extremely powerful X-class flares, fast coronal mass ejections (CMEs), solar energetic particle (SEP) events and so on. The commonly accepted idea is that each of the powerful solar eruptive events in the Halloween epoch of 2003 was related to a single AR, e.g., AR 10484, 10486, or 10488; the contribution of the larger magnetic topologies where an AR located was negligible.

Zhang et al. (2007) studied the global magnetic connectivity in CME associated dimmings in one of the major event on October 28. They find that though the CME-related flare happened in one AR, e.g., 10486, the CME-related coronal dimming spread across multiple magnetic topologies as shown in Figure 1a-1c. Brightening and dimming appear in both hemisphere, in ARs as well as under large-scale arcades (see Fig. 1(c)). The background image of Figure 1d is a part of MDI image at 12:48 UT on 28 Oct. 2003, in which eight quasi-simultaneously emerged EFRs are outlined by the pink lines. These EFRs do locate in the places with key magnetic connectivity associated with brightening and dimming. It has been proposed that a large-scale inter-coupled magnetic flux system built a magnetic connection among the different ARs, as well as, some large-scale magnetic structures (Zhou et al. 2007). The instability in the interactive multi-flux system is believed to be responsible for the generation of this and other flares/CMEs during this period. It supports the idea of some sub-photospheric link and global activity on the Sun for this Halloween epoch. These results show that the instability of the large or global magnetic structure, in which there was vigorous interaction of multi-scale flux system, would be the true origin of the prolific CME outbursts. 


\subsection{On the Bastille Day event in 2000}

Bastille day flare/CME has been well studied. Wang et al. (2006) have demonstrated that this major event is not related to only one single AR, but the activation and eruption of a huge transequatorial filament are seen to precede the simultaneous filament eruption and flare in AR 9077, and the full halo-CME in the high corona. Wen et al. (2006) illustrate the wide spread of non-thermal radio bursts, i.e., the non-thermal electron sources, based on the NRH (Nancay radio-heliograph) data. The non-thermal electron sources cover almost the full range of longitude and wide range of latitude of the visible hemisphere. Radio Bursts 'B' and 'E' (see Fig. 2) are spatially correlated with the magnetic structure of the transequatorial filament. Moreover, the non-thermal electron sources spread more broadly than the EUV waves shown by SOHO/EIT observations.
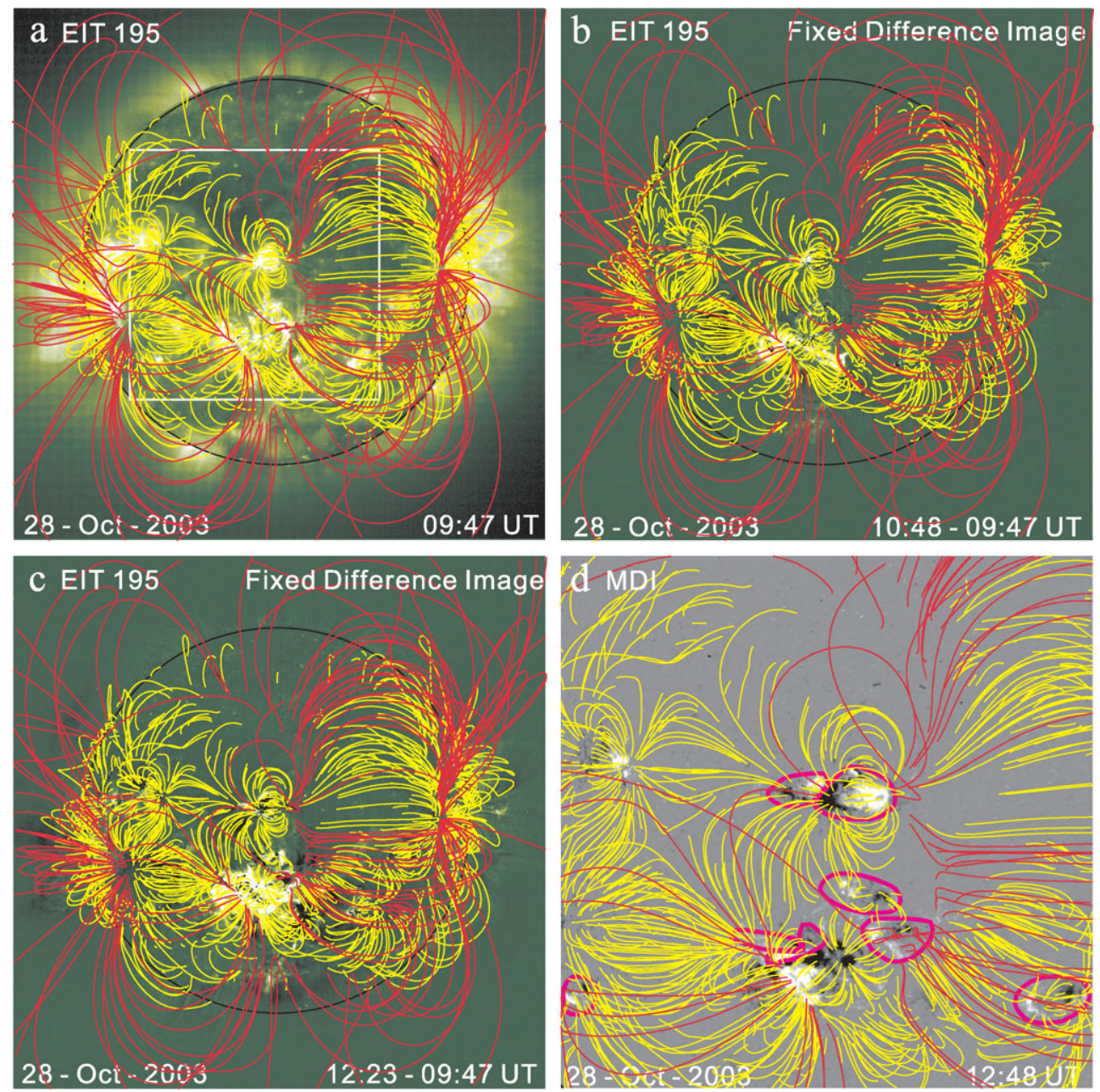

Figure 1. Global magnetic connectivity is found in the CME-associated dimmings and quasi-simultaneous magnetic flux emergence on Oct. 28 2003. Potential extrapolation of observed magnetic fields are overlaid on different background images, i.e., an EIT image at 09:47 UT (a), EIT fixed difference images from 10:48-09:47 UT (b) and from 12:23-09:47 UT (c). Panel (d) is MDI image at 12:48 UT, whose size is indicated by the white square in Panel (a). The contours in pink color denote eight quasi-simultaneous emerging flux regions (EFRs) related to the super activity. 


\subsection{On the events in early November of 2004}

The events in the early November of 2004 are characterized by vigorous activity in transequatorial loops (TELs) and trans-equatorial filaments (TEFs) (Wang et al. 2007). A set of TELs are connecting opposite polarity flux of AR10696 \& 695 plus a coronal hole on both hemispheres and laying above the large-scale trans-equatorial magnetic neutral lines, above which there appear an active trans-equatorial filament since November 7 , in the interval of November 3-9. During the eruption of the trans-equatorial filament, a beautiful two-ribbon flare was shown by SOHO/EIT $19.5 \mathrm{~nm}$ images (also shown by $\mathrm{H} \alpha$
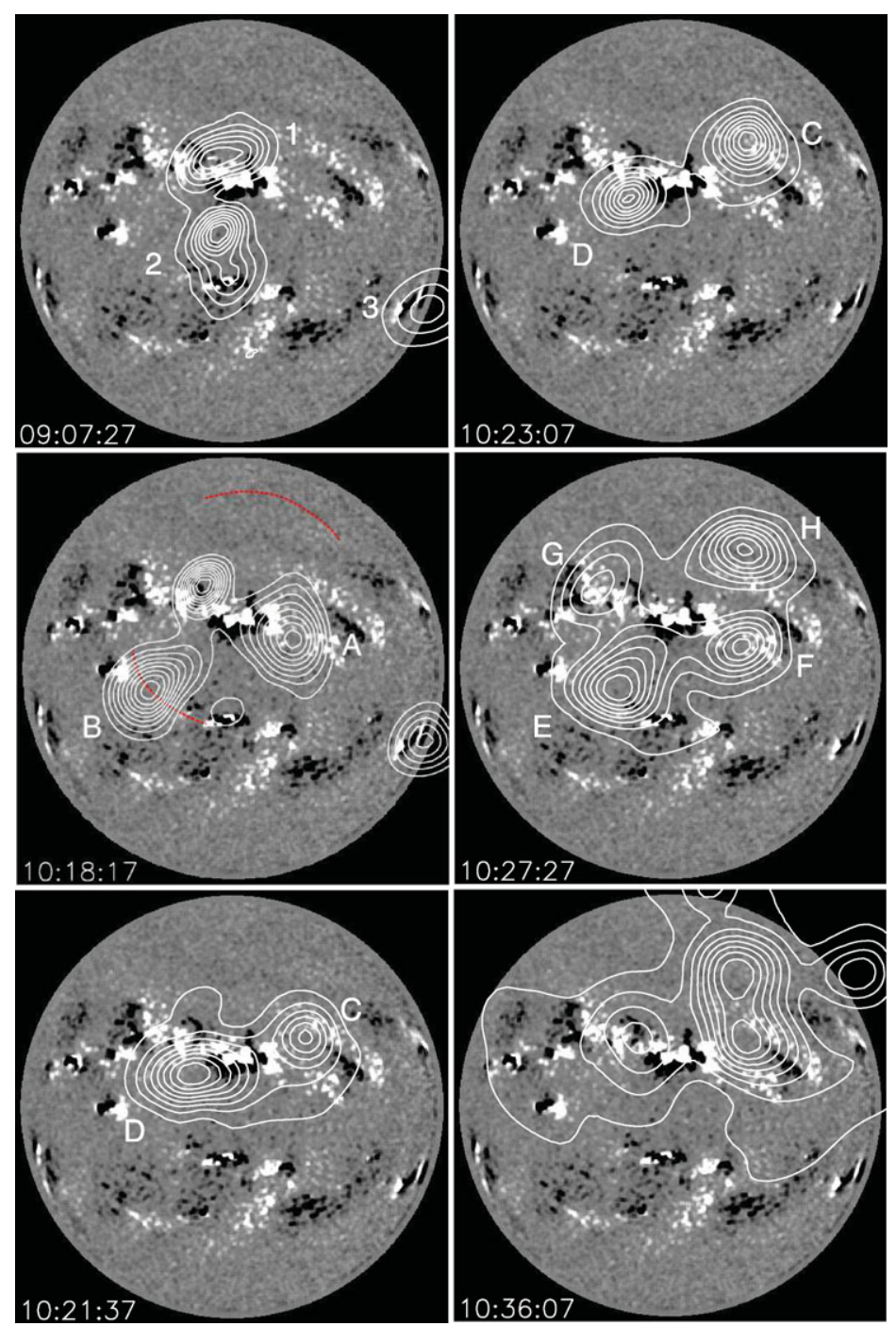

Figure 2. Radio emission sources at $164 \mathrm{MHz}$ obtained from $\mathrm{NRH}$ superposed on the MDI magnetograms at the closest time. Pre-event Type I storm sources are numbered as '1', '2', '3', and burst sources are denoted by capital letters (see Wen el al. 2006). The wide spreading of non-thermal electron source is shown during CMEs, which show that large-scale magnetic systems were involved in the CME development. The EIT wave front at 10:18 UT is denoted by two red lines in the middle left panel. The angular resolution of the NRH is $5.5^{\prime} \times 3.2^{\prime}$ at $164 \mathrm{MHz}$. 
images, but much more diffuse), which overran the size of AR10696 (See Fig. 8 of Wang et al. 2007). Often, a TEL is growing and flaring, coupled with major flare or flares in AR 10696, during which the associated CME was accelerating. The energy release rate of a TEL is much lower than that of a major flare in the AR, but their lifetime was longer than 15 hours (see Figs. 3 and 11 of Wang et al. 2007). Jiang et al. (2007) have revealed from a theoretical approach that TELs are visible signatures of poloidal field lines across the equator in Babcock-Lighton dynamo process, and an intrinsic component of solar magnetism in solar cycle. However, as an AR not always produces flares, only episodic flaring happens in some TELs.

\section{Discussions}

The observations, as those described above, support an idea that some CMEs with wide-span may have their source regions embedded in large- or global-scale magnetic structures, though their initiation site is in an AR. A single active region with its flare activity may not be capable of producing a CME with large angular width. Zhukov and Veselovsky (2007) suggest a concept of global CMEs to describe observations of widespan CMEs whose source regions are truly global in nature. Activity with large spatial scale have been clearly shown in trans-equatorial magnetic structures (Harra et al. 2003, 2007; Wang et al. 2006, 2007).

Driel-Gesztelyi and her co-authors (2008) suggest on the other hand that the wide-span CMEs, or large- or global-scale activity events, initiate from small scale then become large in the low corona by successive interaction of expanding structures of CME itself with surrounding magnetic structures. For the same events in the 2003 Halloween epoch, they drawn a nice cartoon to illustrate their idea (see their Fig. 12). One possible problem with their cartoon is in the fact that whenever there are multi-polar magnetic configuration, nulls develop and a topology skeleton, e.g., spines, fans, and null-null lines, develop and couple the multi-flux systems (see, Zhao et al. 2008). A least-complex configuration which show the basic property of multi-polar magnetic complexity is a quadrupole flux system. MHD numerical simulation shows that for such a multi-flux system, double current sheets develop and catastrophes happen immediately (Zhang et al. 2006; Zhang \& Wang 2007) to respond the magnetic evolution in the photosphere, so that the magnetic breakout takes place on the current sheet above the global flux system. The magnetic break-out model (Antiochos 1998, Antiochos et al. 1999) appears to be a model that relies on or comprise a large or global-scale magnetic source for CMEs.

On the contrary of the idea of large scale CME source, Moore et al. (2007) argued that the final angular width of a CME can be directly related to the flaring AR. It might be true for their examples. However, in pretty rich literatures, large-scale CME sources are shown (see Wang et al. 2002). Zhou et al. (2006) find that about $40 \%$ of earth-directed CMEs are associated with trans-equatorial loops.

However, taking the study of TELs as an example, we still have not fully understand the physics in many aspects, e.g., TEL's footprints in magnetic clouds. For quite long time, our solar astronomers seem to concentrate ourselves on AR and flare activity; on the other hand, the full disk observations with adequate cadence and temporal coverage are still not enough. Future diagnosis of more global magnetism and activity is fundamental in understanding solar and stellar activity.

\section{References}

Antiochos, S. K. 1998, ApJ, 502, L181-L184

Antiochos, S. K., DeVore, C. R., \& Klimchuk, J. A. 1999, ApJ, 510, 485-493 
Attrill, G., Nakwacki, M. S., Harra, L. K., van Driel-Gesztelyi, L., Mandrini, C. H., Dasso, S., \& Wang, J. 2006, Sol. Phys., 238, 117-139

Attrill, G., Harra, L. K., van Driel-Gesztelyi, L., \& Demoulin, P. 2007a, ApJL, 656, L101-L104

Bemporad, A., Sterling, A. C., Moore, R. L., \& Poletto, G. 2005, ApJL, 635, L189-L192

Culhane, J. L. \& Siscoe, G. L. 2007, Sol. Phys., 244, 3-12

Harra, L. K., Matthews, S. A., \& van Driel-Gesztelyi, L. 2003, ApJ, 598, L59-L62.

Harra, L. K., Crooker, N. U., Mandrini, C. H., van Driel- Gesztelyi, L., Dasso, S., Wang, J., Elliott, H., Attrill, G., Jackson, B. V., \& Bisi, M. M. 2007a, Sol. Phys., 244, 95-114

Harra, L. K., Hara, H., Imada, S., Young, P. R., Williams, D., Sterling, A., Korendyke, C., \& Attrill, G. 2006, Publ. Astron. Soc. Japan, 59, S801-S806

Liu, Y. 2007, ApJ, 654, L171-L174

Maia, D., Vourlidas, A., Pick, M., Howard, R., Schwenn, R., \& Magalhães, A. 1999, J. Geophys. Res., 104, 12507-12514

Mandrini, C. H., Nakwacki, M. S., Attrill, G., van Driel-Gesztelyi, L., Demoulin, P. Dasso, S., \& Elliot, H. 2007, Sol. Phys., 244, 25-43

Moore, R., Sterling, A. C., \& Suess, S. T. 2007, ApJ, 668, 1221-1231

Pohjolainen, S., Maia, D., Pick, M., Vilmer, N., Khan, J. I., Otruba, W., Warmuth, A., Benz, A., Alissandrakis, C., \& Thompson, B. J. 2001, Astrophys. J., 556, 421-431

van Driel-Gesztelyi, L., Attrill1, G. D. R., Démoulin, P., Mandrini, C. H., \& Harra, L. K. 2008, Ann. Geophys., 26, 3077-3088

Wang, J., Zhou, G., Wen, Y., Zhang, Y., Wang, H., Deng, Y., Zhang, J., \& Harra, L. K. 2006, ChJAA, 6, 247-259

Wang, J.-X., Zhang, Y.-Z., Zhou, G.-P., Harra, L.,Williams, D., \& Jiang, Y.-C. 2007, Sol. Phys., $244,75-94$

Wang, T., Yan, Y., Wang, J., Kurokawa, H., \& Shibata, K. 2002, ApJ, 572, 580-597

Wen, Y., Wang, J., Maia, D. J. F., Zhang, Y., Zhao, H., \& Zhou, G. 2006, Sol. Phys., 239, $257-276$

Zhang, Y., Wang, J., Attrill, G. D. R., Harra, L. K., Yang, Z., \& He, X. 2007, Sol. Phys., 241, $329-349$

Zhang, Y., Wang, J., \& Hu, Y. Q. 2006, ApJ, 641, 572-576

Zhang, Y. \& Wang, J. 2007, ApJ, 663, 592-597

Zhao, H., Wang, J., Zhang, J., Xiao, C.-J., \& Wang, H. 2008, ChJAA, 8, 133-145

Zhou, G.-P., Wang, J.-X., Wang, Y.-M., \& Zhang, Y.-Z. 2007, Sol. Phys., 244, 13-24

Zhukov, A. N. \& Veselovsky, I. S. 2007, ApJL, 664, 131-134

Zirin, H. 1985, Aust J. Phys., 38, 961-969 